\title{
Smart Data as a Service
}

\author{
Thang Le Dinh ${ }^{1, *}$ and Nguyen Anh Khoa Dam ${ }^{1}$ \\ ${ }^{1}$ Business School, Université du Québec à Trois-Rivières, C.P 500, Trois-Rivières, Québec, G9A5H7, \\ Canada
}

\begin{abstract}
Nowadays, smart data emerge as a new research direction to create value from business data in an intelligent way. Smart data are defined as the data gathered and processed that can be used to create new insights for smart solutions to support business strategies. This paper aims at proposing a conceptual model for smart data management. In other words, the model can be used for designing a smart service system based on the perspective of service science that can manage and deliver smart data as a service.
\end{abstract}

\section{Introduction}

Nowadays, the new development of big data, business analytics, and artificial intelligence has fundamentally changed traditional business processes $[1,2]$. Enterprises are under pressure to innovate and create unique and exceptional competitive advantages. One of the most important challenges faced by enterprises is how to create value from business data, especially big data $[3,4]$. Smart data are defined as the data gathered and processed that can be used to generate new insights for smart solutions to support business strategies $[5,6]$.

This paper aims at expanding knowledge regarding the management of smart data in today's business landscape in order to develop new intelligence of smart data and solutions in the era of big data and artificial intelligence. Smart solutions, which are built based on smart data and intelligent systems and services, have the capacity of self-detecting and self-adaptation to users' needs without their explicit requests [5, 7]. Big data, business analytics, the Internet of Things, and cloud computing provide a huge source of knowledge that needs to be transformed into smart data, to determine user contexts, and then to enable intelligence capabilities of smart solutions $[5,8]$.

However, there is still a little focus on how to transform big data into a higher level of data that can be used for smart solutions [6,9]. For this reason, this paper aims at proposing a conceptual model for designing a smart service system, which can manage and deliver smart data as a service. Based on the service science perspective, smart data management is an emerging research direction that concerns the management, the science, and the engineering of smart data [10-12].

The paper is structured as follows. Section 2 continues with the principles of smart data. Section 3 presents the actionable insights and the challenges of the transformation from smart data to actionable insights. Section 4 proposes the conceptual model for smart data management and Section 5 ends with the conclusion and future work.

\footnotetext{
${ }^{*}$ Corresponding author: thang.ledinh@uqtr.ca
} 


\section{Smart data}

Enterprises are overwhelmed with big data; however, big data are not important if no insights are extracted $[12,13]$. In a sense, smart data mean the "right data" that can reveal insights and make these insights actionable. Smart data are the combination of big data and deep data $[8,14]$. Deep data can provide the context and calibration of a researched phenomenon that is a challenge of big data $[12,14]$. To put it another way, big data are perceived as smart data when they can generate meaningful insights and create value [5]. Analytics transforms meaningless numbers into actionable insights. From the perspective of analytic techniques, patterns and insights of smart data are extracted by intelligent algorithms [6]. With the support of state-ofart analytics, smart data can be generated at the point of data collection [8]. Therefore, enterprises can reduce costs for data storage [3]. The real-time attribute of smart data also leverages the value of big data through various decision supports [4, 14]. Building from these reflections, this study defines smart data as a subset of big data that can provide actionable insights through the process of analytics [5, 6, 12].

In terms of significance, smart data respond to the challenges of big data that most enterprises encounter [6]. In particular, smart data deal with the problems of data overload and data quality due to the characteristics of big data such as huge volume, velocity, veracity, and variety $[9,10]$. In accordance with this view, the study of García, Ramírez-Gallego, Luengo, Benítez and Herrera [11] defines smart data as an important step of data preprocessing to provide a smart dataset in a timely and accurate manner. Accordingly, adopting smart data helps enterprises determine the most current and relevant data sources as all data sources are not equal [15]. Actually, focusing on the right data source can improve the quality of data.

\section{From smart data to actionable insights}

As discussed in the previous part, smart data outperform big data as they provide actionable insights $[5,6,12]$. Actionable insights are defined as meaningful findings through the process of data analytics. Enterprises rely on actionable insights for data-driven decisions [18]. With the supports of actionable insights, enterprises are enlightened with actions that need to be taken in dealing with complex business situations $[6,19]$.

Not all insights are actionable [6]. Insights are actionable in the sense that enterprises can draw conclusions and take actions upon business situations $[18,20]$. In fact, about $70 \%$ of enterprises struggle with taking data analytics to the next step for action plans [21].

In other words, actionable insights are the gap between data and business value [22]. This stimulates the motivation to clarify factors that support actionable insights. Firstly, actionable insights should be aligned with the goals and strategies of an enterprise to drive actions [23]. Secondly, insights are actionable by being aware of the context or circumstances of service providers (e.g.: organizational culture, strategies, capacities) and customers (e.g.: time, location, preference, etc.) $[18,24]$. Accordingly, a service solution can be recommended to the right customer at the right time in the right setting $[1,10]$. Finally, actionable insights should be specific, clear, critical, and innovative so that decision-makers are stimulated to act upon them $[25,26]$. These characteristics of actionable insights make enterprises comprehensively understand an insight, its importance, priority, and feasibility [27]. The literature points out that insights are not well manifested towards models or business rules/processes [19, 28]. Actionable insights need to be visualized through digital dashboards or models to support the decisionmaking process $[20,29]$. 


\section{IESS 2.1}

\section{Conceptual model for smart data management}

This section presents a conceptual model for smart data management that set the foundation for designing a smart service system from the perspective of service science. A smart service system is a context-aware service system, which can dynamically adapt to a context and support the decision-making process for a specific business situation [32]. This perspective comprises three elements: science, management, and engineering [30].

Figure 1 illustrates the conceptual model for smart data management, including the engineering, science, and management elements.

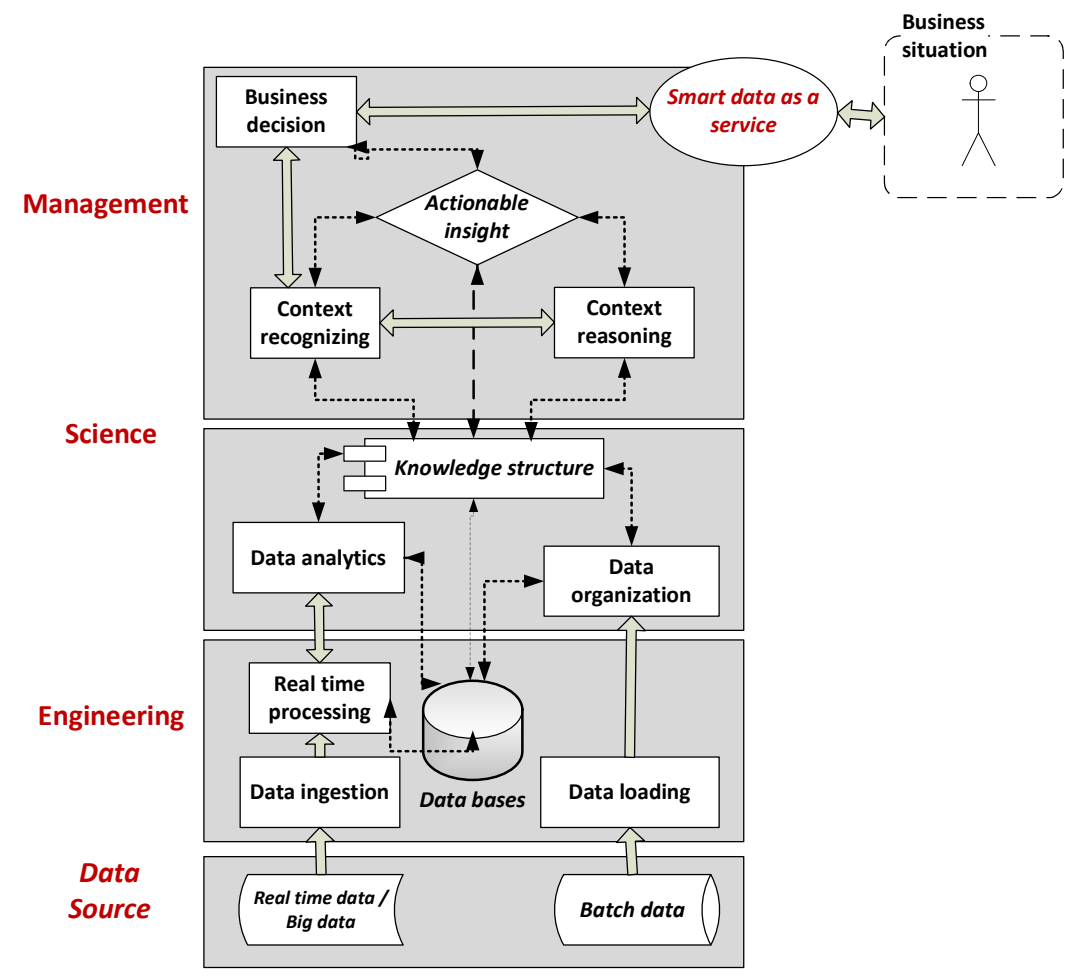

Fig. 1. Conceptual model for smart data management.

Engineering element. The engineering element, which aims at capturing different types of data, covers the invention of new technologies to obtain big data and deep data from different data sources and transform them into useful data stored in database management systems [30]. The new sources of data and techniques for data capturing can improve the quality of business services and create new innovative services related to smart data. This element includes components such as data loading, data ingestion, and real-time processing components to process different data sources to support data collection, provision, and distribution model [31].

Science element. The science element, which focuses on organizing data into useful information, deals with the structure of service systems and facilitates the process of service creation and the application of competencies [30]. A knowledge structure is defined as an interrelated collection of concepts of a domain, relationships between concepts, and relationships between concepts and a data source. In our approach, concepts are defined by different knowledge components such as know-what, know-how, know-why, know-where, know-when, know-who, and know-with [31,32]. The data analytics and data organization 
components help to discover new types of data and knowledge, to link data sources with relevant concepts and determine relationships between concepts.

Management element. The management element, which aims at transforming useful information into actionable insights, concerns methods and techniques to improve services related to smart data through effective management [30]. The objectives of this element focus on the control, discovery, collaboration, learning, and decision support based on actionable insights [31]. Smart data as a service provide a service to a decision-maker based on a particular business situation. The context recognizing and context reasoning components help the business decision component to determine the context of the corresponding business situation. A context is defined as "a stakeholder (know-who) performs actions (know-how) on objects (know-what) at a certain time (know-when) in a location (know-where) because of a contract (know-with) to be consistent with a business rule (know-why)" [32]. Thus, the business decision component may provide the possible solutions and recommend a specific solution based on business intelligence and analytics techniques performing on actionable insights and related data sources.

\section{Conclusion}

This paper proposes a conceptual model for designing a smart service system based on the perspective of service science that can manage and deliver smart data as a service. It is believed that this study is one of the first that focuses on supporting smart data management from the service science perspective.

Concerning the implications of our work for practice, the proposed model sets a strong foundation for change management on smart data and for organizational adaptation on business structure and systems to support smart data. In fact, smart data also facilitate the need for managerial, organizational, and technological changes corresponding to the management, science, and engineering elements of the model [10-12]. The management changes focus on developing business strategies to offer context-aware smart services $[16,17]$. The organizational changes address the significance of organizational culture, structure, business process, and leadership for smart data management $[5,7]$. The technological changes emphasize the need for automation tools for collecting and transforming big data and deep data for smart data capture $[8,10]$. In summary, the ultimate importance of smart data is its transformation of enterprises that struggle with data into data-driven enterprises for smart solutions [3, 4].

Concerning the implications of our work for research, the proposed model can be a starting point for studies on smart data management and application in business. We are currently developing the framework for smart data management based on our previous work [32] under the informational lights of service science for the progression [33].

\section{References}

1. Z. Liu, R. Kettimuthu, I. Foster, P.H. Beckman, Toward a smart data transfer node, Future Generation Computer Systems 89: 10-18 (2018)

2. C. Tan, Y. Sun, G. Li, G. Jiang, D. Chen, H. Liu, Research on gesture recognition of smart data fusion features in the IoT, Neural Computing and Applications, 1-13 (2019)

3. A. Lenk, L. Bonorden, A. Hellmanns, N. Roedder, S. Jaehnichen, Towards a taxonomy of standards in smart data, in IEEE International Conference on Big Data (Big Data), IEEE, 1749-1754 (2015) 
4. M.H. Alsharif, A.H. Kelechi, K. Yahya, S.A. Chaudhry, Machine learning algorithms for smart data analysis in internet of things environment: taxonomies and research trends, Symmetry 12(1), 88 (2020)

5. F. Iafrate, From big data to smart data, John Wiley \& Sons (2015)

6. I. Triguero, D. García-Gil, J. Maillo, J. Luengo, S. García, F. Herrera, Transforming big data into smart data: An insight on the use of the k-nearest neighbors algorithm to obtain quality data, Wiley Interdisciplinary Reviews: Data Mining and Knowledge Discovery 9(2), e1289 (2019)

7. J. Edelenbos, F. Hirzalla, L. van Zoonen, J. van Dalen, G. Bouma, A. Slob, A. Woestenburg, Governing the complexity of smart data cities: Setting a research agenda, Smart Technologies for Smart Governments, Springer 2018, 35-54

8. N. Dey, S. Wagh, P.N. Mahalle, M.S. Pathan, Applied machine learning for smart data analysis, CRC Press, 2019

9. U. Sivarajah, M.M. Kamal, Z. Irani, V. Weerakkody, Critical analysis of Big Data challenges and analytical methods, Journal of Business Research 70: 263-286 (2017)

10. A. Sheth, Transforming big data into smart data: Deriving value via harnessing volume, variety, and velocity using semantic techniques and technologies, in IEEE 30th International Conference on Data Engineering (ICDE), IEEE Computer Society, (2014)

11. S. García, S. Ramírez-Gallego, J. Luengo, J.M. Benítez, F. Herrera, Big data preprocessing: methods and prospects, Big Data Analytics 1(1): 1-22 (2016)

12. K.A. Whitler, Stop Focusing On Big Data And Start Focusing On Smart Data, Forbes, (2019)

13. J. Chen, D. Dosyn, V. Lytvyn, A. Sachenko, Smart data integration by goal driven ontology learning, in INNS Conference on Big Data, Springer, 283-292 (2016)

14. S.V. Kalinin, B.G. Sumpter, R.K. Archibald, Big-deep-smart data in imaging for guiding materials design, Nature Materials 14(10): 973-980 (2015)

15. D. García-Gil, J. Luengo, S. García, F. Herrera, Enabling smart data: noise filtering in big data classification, Information Sciences, 479, 135-152 (2019)

16. N. Roedder, D. Dauer, K. Laubis, P. Karaenke, C. Weinhardt, The digital transformation and smart data analytics: An overview of enabling developments and application areas, in IEEE International Conference on Big Data (Big Data), 27952802 (2016)

17. M. Rakhra, R. Singh, Smart data in innovative farming, in Materials Today: Proceedings, (2021)

18. D. Delen, S. Ram, Research challenges and opportunities in business analytics, Journal of Business Analytics 1(1): 2-12 (2018)

19. A. Syamsiyah, A. Bolt, L. Cheng, B.F. Hompes, R.J.C. Bose, B.F. van Dongen, W.M. van der Aalst, Business process comparison: A methodology and case study, in International Conference on Business Information Systems, Springer, 253-267 (2017)

20. M. Murphy, J. Barton, From a Sea of Data to Actionable Insights, (2014)

21. B. Dykes, Actionable insights: The missing link between data and business value, Forbes, (2016)

22. J. Shim, R. Taylor, Purchase-Based Analytics and Big Data for Actionable Insights, IT Professional 21(5): 48-56 (2019)

23. S. Gupta, A. Leszkiewicz, V. Kumar, T. Bijmolt, D. Potapov, Digital analytics: Modeling for insights and new methods, Journal of Interactive Marketing 51: 26-43 (2020) 
24. W. He, X. Tian, Y. Chen, D. Chong, Actionable social media competitive analytics for understanding customer experiences, Journal of Computer Information Systems 56(2): 145-155 (2016)

25. A. Marshall, S. Mueck, R. Shockley, How leading organizations use big data and analytics to innovate, Strategy \& Leadership, (2015)

26. J. Hester, T.R. Miller, J. Gregory, R. Kirchain, Actionable insights with less data: guiding early building design decisions with streamlined probabilistic life cycle assessment, The International Journal of Life Cycle Assessment 23(10): 1903-1915 (2018)

27. R. McCreadie, C. Buntain, I. Soboroff, Incident Streams 2019: Actionable Insights and How to Find Them, in Proceedings of the International ISCRAM Conference, (2020)

28. A. Levenson, A. Fink, Human capital analytics: too much data and analysis, not enough models and business insights, Journal of Organizational Effectiveness: People and Performance, (2017)

29. V.S. Sharma, R. Mehra, S. Podder, A.P. Burden, A journey towards providing intelligence and actionable insights to development teams in software delivery, in the 34th IEEE/ACM International Conference on Automated Software Engineering (ASE), 1214-1215 (2019)

30. T. Le Dinh, T. T. Pham Thi, Information-driven framework for collaborative business service modelling, International Journal of Service Science, Management, Engineering, and Technology (IJSSMET) 3(1): 1-18 (2012)

31. T. Le Dinh, T. C. Phan, T. Bui, M.C. Vu, Towards a Service-Oriented architecture for knowledge management in big data era, International Journal of Intelligent Information Technologies (IJIIT) 14(4): 24-38, (2018)

32. T. Le Dinh, T. T. Pham Thi, C. Pham-Nguyen, Towards a Context-Aware Knowledge Model for Smart Service Systems, In International Conference on Computational Collective Intelligence, Springer (2020)

33. M. Léonard, Informational Lights from Service Science for the progression of Society (EDP Sciences, Les Ulis, 2020) 